\title{
Valeurs olympiques et réseaux sociaux numériques : controverses et utopies
}

Olympic values and digital social networks: controversies and utopias

\section{Faika Ouergli, Franck Debos et Vincent Meyer}

\section{(2) OpenEdition}

\section{Journals}

Édition électronique

URL : http://journals.openedition.org/communicationorganisation/5072

DOI : 10.4000/communicationorganisation. 5072

ISSN : $1775-3546$

Éditeur

Presses universitaires de Bordeaux

\section{Édition imprimée}

Date de publication : 1 décembre 2015

Pagination : 73-92

ISSN : 1168-5549

\section{Référence électronique}

Faika Ouergli, Franck Debos et Vincent Meyer, «Valeurs olympiques et réseaux sociaux numériques: controverses et utopies », Communication et organisation [En ligne], 48 | 2015, mis en ligne le 01 décembre 2018, consulté le 04 janvier 2020. URL : http://journals.openedition.org/

communicationorganisation/5072 ; DOI : 10.4000/communicationorganisation.5072 


\title{
Valeurs olympiques et réseaux sociaux numériques: controverses et utopies
}

\author{
Faika Overgli ${ }^{1}$, Franck Debos ${ }^{2}$ et Vincent Meyer ${ }^{3}$
}

Le propos s'appuie sur deux constats. Le premier est la faible présence et visibilité des valeurs de l'olympisme dans la communication lato sensu des instances sportives au plan national et international alors que l'on s'attendrait à ce qu'elles occupent une place significative dans leurs stratégies de communication notamment numériques en direction du grand public. Le second, alors que nombre de scandales divers et de dopage sont dévoilés et entachent le mouvement sportif, des communautés virtuelles réagissent - en controverse - sur l'absence de référence aux valeurs de l'olympisme. La situation pourrait dès lors tenir du paradoxe : les organisations sportives peinent à afficher des références aux valeurs olympiques et des communautés virtuelles en débattent et s'y substituent. Assurément, si la symbolique du sport et de l'olympisme s'est élaborée autour de prouesses, de performances et d'une compétition entre les individus, elle se traduit aussi par des représentations idéalisées et partagées par les dirigeants, les pratiquants, les médias, les pouvoirs publics (Diana, Meyer 2004). Les spectateurs du monde sportif dans leurs mises en réseaux véhiculent à leur tour pareilles représentations et les associent communément au sport avec différents corollaires dont la morale et l'éthique que l'on devrait (re)trouver au cœur des interactions numériques entre pratiquants et institutions sportives.

\footnotetext{
1 Faika Ouergli est Docteure en sciences de l'information et de la communication de l'université Nice Sophia Antipolis ; ses domaines de recherche sont les valeurs olympiques, le marketing du sport, le e-marketing et les réseaux sociaux. Elle étudie le comportement de l'internaute et les intentions de la communication en ligne ; faikaouergli@yahoo.fr

2 Franck Debos est Chercheur au laboratoire Information, milieux, médias, Médiations (I3M EA 3840) et Maître de conférences à l'IUT de Nice Côte d'Azur, ses domaines de recherche, d'enseignement et de consultant concernent la communication responsable et numérique des organisations, le marketing, la responsabilité sociale de l'entreprise ainsi que l'analyse des usages des consommateurs; debos.franck@wanadoo.fr

3 Vincent Meyer est Professeur des universités en sciences de l'information et de la communication à l'université Nice Sophia Antipolis et chercheur au laboratoire Information, milieux, médias, Médiations (I3M EA 3840); ses domaines de recherche sont les pratiques d'information et de communication d'agents individuels ou collectifs dans différents champs professionnels et leur traduction sur des supports protéiformes (www.vmeyer. canalblog.com); Vincent.Meyer57@wanadoo.fr
} 
Nous nous situons dans une situation de controverse puisque différents acteurs dans le domaine sportif vont être en conflit sur plusieurs questions et enjeux autour des valeurs de l'olympisme. Il s'agit de présenter une cartographie de cette controverse, telle que définie par Bruno Latour (2007) à savoir, l'analyse empirique d'une situation contemporaine caractérisée par des oppositions entre des groupes d'acteurs. Pour qualifier cette controverse, cette recherche convoque une analyse de l'architecture et du contenu rédactionnel de sites institutionnels du sport couplée à un recueil de données en ligne permettant de saisir les discours des membres d'une communauté créée sur Facebook et regroupant des personnes ayant une pratique sportive régulière à un niveau professionnel ou amateur. Les perspectives ainsi ouvertes sont aussi opérationnelles, i.e. proposer des orientations stratégiques permettant aux organisations sportives de promouvoir les valeurs de l'olympisme par le biais des technologies numériques. Ceci est d'autant plus important que cette controverse va au-delà d'une coproduction d'un discours symbolique sur des valeurs dites universelles. Elle montre que si elles sont devenues sinon incommunicables tout du moins ambiguës dans le contexte actuel, des communautés virtuelles adhèrent, elles, à une représentation vertueuse du sport olympique qui leur apparaît comme une mesure protectrice du mouvement sportif face à ses dérives. In fine cette recherche interroge à nouveaux frais les relations entre différents publics et les organisations sportives via les réseaux sociaux et, plus particulièrement, dans la diffusion des valeurs de l'olympisme. Elle permet également de mettre en débat un modèle "utopien » qui affleurerait avec elles. En effet, les organisations sportives doivent prendre en considération le fait que leurs adhérents ou sympathisants ont le sentiment que ces valeurs universelles ne sont plus ou peu respectées et correspondent à une vision utopique et idéalisée du sport. Deux parties structurent le propos : après avoir rappelé les fondements de l'olympisme dont l'objectif ultime est de placer le sport au service du développement harmonieux de l'humanité pour favoriser une société pacifique engagée au maintien de la dignité humaine (Corral et al. 2010) nous tenterons de montrer que les valeurs associées à l'olympisme - censées représenter "la colonne vertébrale ", non seulement de l'organisation olympique, mais de toute institution structurant le secteur du sport - peuvent être considérées, en première analyse, comme les facettes de l'archétype d'une organisation utopienne. Ceci en deux temps : sérier et mettre au jour, dans cette organisation, ce que Lucien Sfez (2000) nomme les « marqueurs de l'utopie ». Pour ce dernier, les utopies anciennes ont en commun un certain nombre de traits qui permettent d'appréhender les utopies contemporaines.

- montrer comment - si on retient, assez classiquement, la racine du terme Utopia utilisé par Thomas More (Mulliez 2013) pour nommer son île imaginaire « outopos » i.e. « en aucun lieu » ou encore « lieu du bonheur»-, ne s'applique pas vraiment 
à l'olympisme ou, plus précisément, que ses fondements - une société procurant bonheur et épanouissement aux individus - ne se retrouvent pas frontalement au sein de la gouvernance des institutions sportives.

Nous analyserons ensuite l'architecture et le contenu de certains sites institutionnels en lien avec le comité international olympique couplés aux résultats d'une enquête par questionnaires menée auprès d'une communauté développée sur Facebook autour d'une page intitulée « Promotion des valeurs olympiques ». L'échantillon étant composé de personnes ayant une expérience dans le domaine sportif.

En conclusion, et en troisième analyse, nous reviendrons sur ce besoin de «bonne utopie» (Morin 2015) que, sans doute, les organisations comme les institutions (i.e. celles et ceux qui les représentent) peinent de plus en plus à traduire et qui ne se retrouvent plus, tout à fait, dans les grands discours ou dans une ambition à penser l'universel ouvrant in fine comme le suggère Michel Maffesoli (2015) sur une «normopathie».

\section{Valeurs de l'olympisme : les fondements d'une organisation utopienne ?}

En 1908, Pierre de Coubertin pose les fondements de l'olympisme en tant que doctrine fraternelle mêlant le corps et esprit (Durántez 2001, cité par Corral C. D. et al. 2010). L'olympisme, selon la vision de Pierre de Coubertin, vise donc essentiellement le développement harmonieux des aspects intellectuels, moraux et physiques d'un être humain grâce à la compétition sportive (Segrave, Chu 1981).

Les principes fondamentaux de l'olympisme sont énoncés dans la Charte olympique, dont la première version a été publiée en 1989 (Olympic Charter 2004).Elle constitue également la codification des principes fondamentaux, des règles et des arrêtés adoptés par le Comité International Olympique. Aujourd'hui, la Charte olympique qualifie l'olympisme comme une philosophie de vie qui exalte et combine, dans son ensemble harmonieux, les qualités du corps, la volonté et l'esprit. Son objectif ultime est de placer le sport au service du développement harmonieux de l'humanité et ainsi favoriser une société pacifique engagée au maintien de la dignité humaine (Corral C. D. et al. 2010).

Malgré ses idéaux inclus dans la Charte olympique, l'olympisme n'est pas exempt de critiques suscitant des controverses (Hoberman 1986 ; Simson, Jennings 1991 ; Coakley 2001). La montée des nationalismes, l'implication du contexte géopolitique avec notamment le boycott des Jeux Olympiques (JO) par certains pays, la commercialisation, l'accélération de la professionnalisation des athlètes, la discrimination envers l'origine, le sexe et l'ethnicité dans « l'arène » olympique, les scandales concernant la corruption des membres du CIO représentent quelques-unes des principales critiques contre le Mouvement olympique moderne (Chatziefstathiou, 2005). 


\section{Une controverse contextuelle des représentations de l'olympisme}

Le sport se base sur un socle de valeurs humaines et de fondements éthiques baptisé « valeurs olympiques». Il a pour objectif de former des peuples respectueux et solidaires et même permettre de résoudre des problèmes de violence.

Les valeurs olympiques réunissent les principes fondamentaux qui déterminent le comportement humain dans les activités sportives (Boli, 2008). Actuellement, selon le CIO, l'Olympisme est notamment diffusé à travers trois valeurs clés - excellence, respect et amitié - qui expriment des aspects humains, moraux et de qualité.

\section{L'excellence}

Dans l'idéal olympique, l'excellence exprime le fait de donner le meilleur de soi-même, sur le terrain ou dans la vie quotidienne, sans se mesurer aux autres, pour atteindre avant tout des objectifs personnels avec détermination. Il ne s'agit pas seulement de gagner, mais surtout de participer et de progresser par rapport à ses propres buts.

\section{L'amitié}

Une des priorités du Mouvement olympique est d'œuvrer en faveur du rapprochement et de la compréhension entre les peuples. L'amitié renvoie à la volonté de bâtir un monde meilleur et en paix à travers la solidarité, l'esprit d'équipe, la joie et l'optimisme dans le sport. Les Jeux Olympiques inspirent l'humanité à dépasser les différences d'ordres politique, économique, racial, religieux ou de genre, et forger des amitiés malgré ces différences. Les athlètes expriment l'amitié à travers les liens durables qu'ils tissent avec leurs coéquipiers et leurs concurrents.

\section{Le respect}

Dans l'idéal olympique, le respect constitue le principe éthique qui touche toutes celles et tous ceux qui participent aux programmes olympiques. Elle comprend le respect de soi et de son corps, le respect des autres, des règles et de l'environnement. Elle renvoie au fair-play dont tout athlète doit faire preuve, ainsi qu'à la lutte contre le dopage.

À notre connaissance, l'UEFA est la seule union sportive qui a pu diffuser le respect comme une des valeurs olympiques durant toute la coupe d'Europe 2012. Le projet Respect de l'UEFA EURO 2012 est constitué de quatre points : lutte contre le racisme (Respect de la diversité), amélioration de l'accès pour les supporters handicapés (Respect des différences), promotion de la santé grâce à l'activité physique (Respect de votre santé) et incitation au dialogue interculturel entre les fans et les villes hôtes (Respect de la culture des supporters). 
Ces trois valeurs clés peuvent donc être considérées comme les bases structurelles d'une organisation centrée sur l'épanouissement de l'individu dans le respect des autres et de l'environnement. Le questionnement qui s'ensuit consiste à se demander si ces valeurs peuvent servir de fondements d'une organisation utopienne. Il paraît également important de saisir en quoi les organisations sportives, notamment le CIO et d'autres grandes institutions vont baser leur gouvernance sur ces valeurs. Dans le cas contraire, il semble pertinent de voir par qui et de quelles façons ces valeurs sont reprises, diffusées, discutées et magnifiées ce, notamment au niveau du numérique par le biais de communautés virtuelles.

\section{Valeurs de l'olympisme et marqueurs de l'utopie}

Pour étayer notre point de vue, nous emploierons les " marqueurs de l'utopie » développés par Lucien Sfez. Pour ce dernier, les utopies anciennes ont en commun certains traits qui les identifient en tant que genre de discours et qui permettent d'analyser les utopies contemporaines. À ce niveau Lucien Sfez détermine cinq grandes catégories en précisant que ce nombre est évolutif et que d'autres auteurs en ont identifié davantage (Rasse 2003). Nous tenterons de montrer que la plupart de ces marqueurs se retrouvent dans les valeurs à la base de l'olympisme et le modèle organisationnel qu'elle soustend, modèle que l'on peut assimiler à une organisation utopienne. L'étude de l'olympisme et de ses valeurs met en exergue les marqueurs suivants.

- Un lieu présent partout et nulle part : lorsque Thomas More (14781535) crée le mot utopie, il imagine une île existant nulle part mais pouvant être présente partout. Dans le même esprit lorsque Pierre de Coubertin parle de l'olympisme comme d'une école de noblesse et de pureté morale, cette école idéale n'existe pas réellement mais dans la mesure où elle se fonde sur des valeurs universelles, elle pourrait se retrouver partout. Il y a donc ici une similitude entre l'utopie et l'olympisme.

- Un retour aux origines : Lucien Sfez précise qu'une des constantes des projets utopistes consiste en la quête d'un Eden perdu ou les hommes vivaient dans la paix et l'harmonie. Nous le voyons avec Aristote et sa description de l'âge d'or ou les hommes vivaient un bonheur parfait sous la houlette du titan Chronos. Thomas More, Tommaso Campanella (1568-1639) et Claude Nicolas Ledoux (1736-1806) font référence à la cité idéale de Platon. Pour terminer, Saint-Simon (1760-1825) et Fourier (1772-1837) pensent aux premières communautés chrétiennes lorsqu'ils traitent de l'Eden perdu. Nous pouvons considérer également que les valeurs de l'olympisme font référence à la Grèce antique qui, dans la vision idéalisée de Pierre de Coubertin apparait comme un Eden perdu permettant un développement harmonieux des qualités physiques, intellectuelles et morales de l'individu. Cette caractéristique de l'utopie se retrouve bien au niveau des valeurs olympiques. 
- Un auteur ou créateur charismatique entretenant des rapports ambigus avec le pouvoir : l'ensemble des utopistes, de Platon à Saint-Simon peuvent se retrouver dans l'assertion précédente. Tous ont en effet eu une personnalité hors du commun et des relations tumultueuse avec le pouvoir en place, tantôt ayant des postes importants, tantôt connaissant la disgrâce, l'emprisonnement voire la mort. Avec Pierre de Coubertin nous retrouvons aussi ce marqueur de l'utopie. En effet, nul ne conteste sa forte personnalité et son charisme. Toutefois, si son nom est souvent associé à un idéal de paix, d'égalité entre les humains il est également perçu comme réactionnaire et aura de par son activisme et son « opportunisme » souvent maille à partir avec les instances sportives nationales et certains gouvernements de la troisième République. Cette ambiguïté s'illustre par le fait que même si sa reconnaissance fut internationale, il ne recevra aucune grande distinction française.

- Un projet urbanistique fort : il s'agit d'une constante dans les projets utopistes (la cité de Platon, les 54 villes orthogonales de Thomas More, le Phalanstère de Fourier, etc.). Nous pouvons faire ici un parallèle avec les infrastructures sportives construites par les villes ayant été sélectionnées pour accueillir les JO d'été ou d'hiver qui sont en général la concrétisation de concepts architecturaux avant-gardistes, symbole du dynamisme et de la puissance du pays hôte. Cette dimension urbanistique est donc bien présente dans les activités olympiques.

- La quête d'un ordre évident : la plupart des projets utopiques présentent des modèles d'organisation de la vie sociale détachés d'un pouvoir pyramidal et faisant appel à la raison des hommes permettant ainsi une dynamique et une harmonie de la communauté. Les principes de l'olympisme prônent un modèle fondé sur la joie de l'effort, la valeur éducative et le respect des principes fondamentaux et universels de l'éthique que l'on peut assimiler à un projet utopique.

- Desforces sociales inhérentes: la plupart des utopistes, indépendamment du modèle organisationnel qu'ils préconisent recherchent une force, une dimension qui sera le ciment de leur organisation et qui s'épanouira dans leur cité idéale. Pour Thomas More, il s'agit de la compassion et la bonté naturelle des hommes, pour Campanella ce sera l'amour des autres et de soi. Fourrier définit cette force comme une "attraction passionnée » et Saint-Simon comme « la sympathie naturelle et la fraternité parfaite ». Ce marqueur de l'utopie est également présent dans l'olympisme. En effet, lorsque De Coubertin parle de l'olympisme comme "la religion de l'énergie, le culte de la volonté intensive ", il fait également référence à cette force sociale permettant de rapprocher les hommes, de créer une société pacifique préservant la dignité humaine.

Comme présenté supra, nous trouvons la quasi-totalité des marqueurs de l'utopie au sein du projet olympique et de ses valeurs. Celles-ci peuvent être considérées comme les fondements d'une organisation utopienne veillant à l'épanouissement de l'individu au sein d'une société pacifique. Or, lorsque 
l'on voit le fonctionnement et la gouvernance des institutions sportives en général, notamment celles ayant une dimension internationale. Il suffit de se référer au scandale récent entachant la FIFA depuis le 3 juin 2015 ayant pour origine des pots-de-vin touchés par plusieurs hauts responsables (14 au départ) de cette organisation pour l'attribution des coupes du monde de 1998 et 2010. Nous sommes loin des valeurs fondant l'olympisme. Ces valeurs servent donc de miroir critique au secteur dont elles sont censées être les fondements comme l'est peut-être toute utopie par rapport à la société où elle se développe.

\section{Une exploration menée par l'analyse des sites institutionnels}

Afin d'étayer nos propos nous avons procédé en deux temps. Nous avons analysé le contenu et l'architecture de différents sites institutionnels en lien avec le Comité International Olympique et certains comités olympiques nationaux. Nous avons également créé sur Facebook une page intitulée "Promotion des valeurs olympiques " pour montrer l'attachement du public sportif aux valeurs de l'olympisme et à l'organisation utopienne qu'elle soustend. La population visée par cette recherche est composée de personnes ayant une expertise du domaine sportif.

Étude du référencement et de l'architecture des sites des institutions sportives

Dans un premier temps, nous avons réalisé en mai 2012une étude comparative entre différents sites institutionnels sportifs afin d'identifier la place qu'ils donnent à ces valeurs, ceci, à l'aide de Google et des mots-clés «valeurs olympiques ». Le classement du moteur de recherche positionne en tête de liste dans la première page : le Comité National Olympique (France), suivi par un blog sur l'actualité du marketing sportif (Olympic Torch Relay), le Comité National Olympique Interfédéral (Belge) et, en quatrième position, le Comité International Olympique (Suisse).

Nous avons de même saisi dans la base de données Wikipédia, les termes «valeurs de l'olympisme ». À ce niveau, le Comité National Olympique Français émerge également en première position, accompagné par les Jeux Olympiques, le site officiel de «JO Beijing 2008 ", suivi par le site "Allons en France ».

Nous exposons ici une synthèse des résultats. L'ensemble de l'analyse est présente dans les tableaux des annexes 1 et 2 .

Par l'étude de l'architecture et du contenu des sites évoqués ci-dessus, nous pouvons remarquer que les valeurs de l'Olympisme ne sont jamais mises en relief et qu'il est impossible d'interagir sur ce point, hormis avec le site «Allons en France ». Cette situation peut illustrer le faible intérêt que portent les structures institutionnelles à interagir avec les communautés virtuelles au sujet des valeurs du sport et de l'idéal Olympique alors que la mise en place d'un espace participatif est techniquement simple (création de forums, 
de blogs, développement de liens destinés à communiquer et transférer des messages aux usagers).

Le fait que cette possibilité d'interagir directement soit absente via ces sites institutionnels, nous incite à postuler que le développement de pareille interactivité pourrait éventuellement engendrer un risque (e.g. la réputation des organisations sportives et notamment de celle du CIO). Il semblerait donc que les comités olympiques n'investissent pas dans toutes les fonctionnalités de l'espace numérique et se privent d'un outil permettant de transmettre les informations et de communiquer avec les acteurs du secteur sportif. Certains chercheurs voient dans ces aspects un effondrement de la logique sportive et compétitive des valeurs (Liotard 2004).

\section{Étude d'une communauté associée à la page Facebook « Promotion des valeurs olympiques »}

\section{Questionner sur la promotion des valeurs olympiques}

Un questionnaire a été diffusé par « Google documents » en février et mars 2012 via les courriers électroniques de nos fans. La formulation des questions repose sur des mots ayant un seul sens, simple, précis et compréhensible par nos participants pour qu'ils acceptent d'y répondre et produire une réponse correcte et non biaisée (Malhotra 2004).

Le questionnaire est composé de trois parties ; d'abord, des questions d'ordre général afin de sérier les connaissances de nos fans sur les valeurs olympiques sur le web et les sites qui les évoquent. Ensuite, nous avons essayé de mesurer le degré de participation et d'engagement de nos enquêtés dans la page communautaire Facebook et si l'expérience sportive a une influence sur leur contribution au niveau de cette page. Enfin, nous avons voulu déterminer si nos répondants sont vraiment attirés par cette page et les raisons qui les incitent à la partager et à l'envoyer à leurs amis dans le but de favoriser la fiabilité et la validité des résultats.

\section{L'échantillonnage}

La population visée est composée des fans de notre page Facebook "Promotion des valeurs olympiques ", constituée de 254 individus de différentes nationalités. Notre groupe est varié, mais a une particularité commune, à savoir l'amour du sport. De même, ses membres ont pratiqué une activité sportive durant une période d'au moins 10 ans jusqu'à plus de 25 ans. La taille de notre échantillon s'avère suffisante pour une première analyse sur la perception des valeurs olympiques sur Internet. Cette recherche garde néanmoins un statut exploratoire. 


\section{Taux de réponse et traitement des données}

Comme indiqué supra, le taux de réponses atteint 63,77\% (sur 254 fans - 162 répondants) sachant que parmi elles, il y a des fans qui ne maitrisent pas suffisamment la langue française. Le traitement des données collectées a été réalisé avec le logiciel Sphinx Plus. Le tri croisé et le test Chi2 ont été employés afin de tester différentes relations entre les variables. Il convient de préciser que cet article ne présente que les résultats de l'enquête directement liés aux réflexions et au champ d'étude en lien avec la thématique du présent numéro. En effet, cette étude avait un cadre de recherche plus large car associée à un travail de thèse (Ouergli 2014).

\section{Analyse et interprétation des résultats}

Le premier résultat concerne la perception des valeurs olympiques sur le web. Les répondants estiment que nous sommes dans une société de plus en plus marchande et que, de fait, les valeurs les plus importantes sont commerciales. La majorité des membres évoque que de nos jours, les valeurs olympiques - comme valeurs morales - sont marginalisées. Nous constatons qu'un important nombre de membres considèrent que celles-ci sont peu valorisées par les institutions sportives sur l'Internet. De plus, les structures institutionnelles représentatives de l'olympisme ne semblent pas avoir pris conscience de l'impact d'Internet, notamment des réseaux sociaux, pour promouvoir leurs valeurs. Aucune ne positionne ces valeurs au cœur de leur gouvernance et de fait, de leur stratégie de communication tant interne qu'externe. Les institutions sportives semblent donc pâtir d'une certaine méconnaissance de l'espace numérique en tant que dispositif sociotechnique de transmission d'informations et d'écoute des acteurs impliqués dans ce secteur. Une logique participative s'avérerait pertinente dans le but de communiquer, de transférer des messages aux cibles déterminées, via l'utilisation des réseaux sociaux, la création de forums ou de blogs référencés au sein des moteurs de recherche. La très grande majorité des répondants, soit 97 personnes sur 162 , considèrent que les valeurs olympiques ne sont pas valorisées par les institutions sportives et notamment olympiques sur l'Internet.

Dans un deuxième temps, nous constatons que la dépendance entre la perception et la pratique dans la communauté est très significative avec un test de chic égal à 56,66\%. Tous les enquêtés ont bien perçu le positionnement de la page. Environ les trois quarts de nos fans $(67 \%)$ postent des commentaires sur la page dans la mesure où ils essayent de promouvoir, de valoriser et de mettre en évidence les valeurs olympiques. Le thème suscite pour nos répondants une attention toute particulière. Seuls 23 répondants (14\%) consultent seulement la page. Les autres consultent et commentent dans le même temps.

Le troisième résultat significatif réside dans le fait que 122 répondants sur 162 promettent de relayer les valeurs olympiques sur Internet par le biais 
des réseaux sociaux. La viralité constitue ainsi une solution efficace pour la diffusion et la valorisation de ces valeurs. Quel que soit le site, la grande majorité des personnes, dans tous les cas, estiment que les valeurs olympiques ne sont pas assez mises en avant par les organismes officiels. Nous pouvons en partie l'expliquer dans la mesure où le $\mathrm{CIO}$ - qui a une fonction de référent ne promeut pas ces valeurs comme nous pouvons déjà le constater via leur site.

Pour terminer, nous pouvons noter que parmi les valeurs de l'olympisme le respect arrive en première position de façon extrêmement forte puisque plus de 3 fois citée (94 personnes sur 162); la deuxième valeur est la paix (30) qui par ailleurs n'est pas intégrée par le $\mathrm{CIO}$ en tant que valeur olympique. Nous retrouvons bien plus loin l'amitié et le fair-play. Nous ne trouvons qu'une des trois valeurs citées par le $\mathrm{CIO}$ ce qui tend à montrer l'importance du respect pour les sportifs. Nous pouvons constater que tous nos répondants adhèrent aux valeurs olympiques même ils arrivent à citer d'autres valeurs qui ne sont pas reprises par le CIO.

\section{Résultats complémentaires tendant à confirmer la controverse}

Notre dispositif de recherche ne s'est pas limité à une étude quantitative, mais a intégré en analyse complémentaire des entretiens semi-directifs réalisés auprès de onze membres de cette communauté ainsi qu'une analyse du contenu des discours de l'ensemble des personnes composant cette communauté. Dans les deux cas, l'analyse a été menée à l'aide du logiciel Tropes. Comme indiqué supra, nous ne présenterons ici que les résultats permettant d'étayer la problématique de notre article.

Au niveau de l'analyse des conversations des membres de la communauté virtuelle de notre page Facebook sur la promotion des valeurs olympiques, nous constatons que les valeurs de l'olympisme sont centrales pour les fans et qu'elles sont à l'origine d'une viralité forte de leur part dans une logique de partage de ces valeurs. Plus important, par rapport à la thématique traitée, les valeurs de l'olympisme sont là encore considérées comme fortement non valorisées par les sites institutionnels.

De ce fait, comme l'indique le graphe suivant, la taille et la proximité des items «valeur perçue » et «non valorisé » confirment le fait que les membres de la communauté considèrent que les valeurs de l'olympisme sont peu valorisées comme par ailleurs le fait que l'item « valorisé » est plus éloigné et de taille plus réduite. 


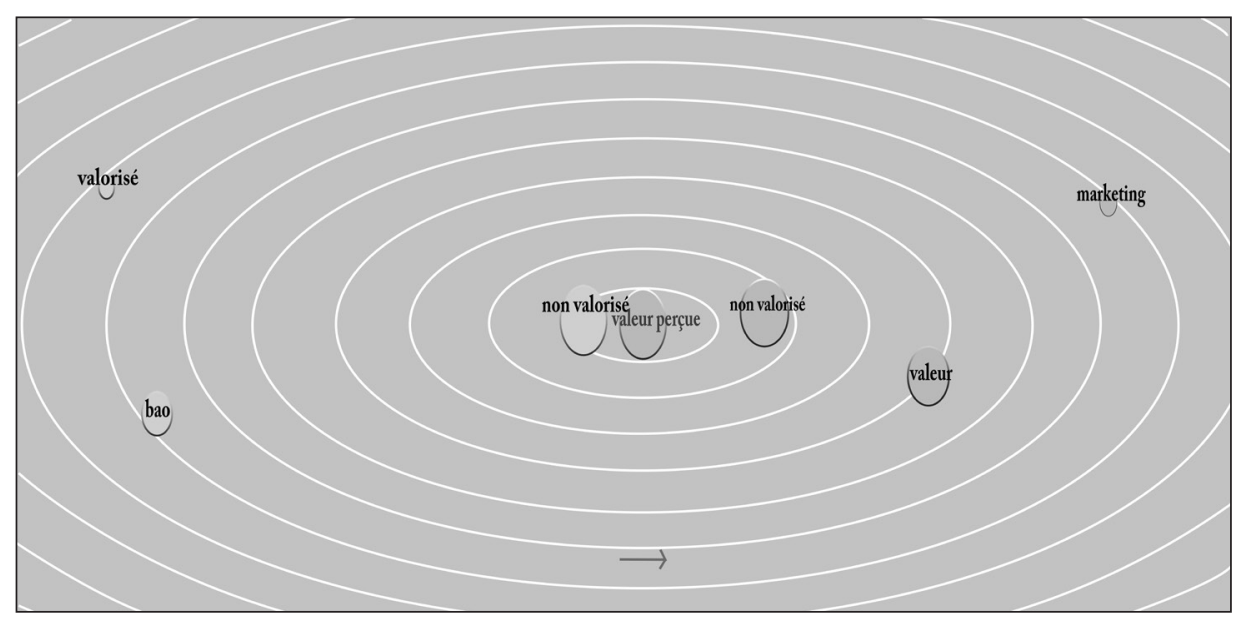

Graphe-Aires (1) : La relation entre valeur perçue, non valorisé et valorisé. Ouergli Faika, 2014, "La promotion des valeurs olympiques au sein des communautés virtuelles : le cas de Facebook $»^{4}$

La place des valeurs de l'olympisme dans les sites officiels est perçue comme très faible par les membres de la communauté créée.

Concernant les entretiens semi-directifs, nous constatons que les «fans" de la page Facebook ont une bonne connaissance des valeurs olympiques comme notamment, le respect qui se trouve en première position (citée à $72,1 \%)$, suivi par l'amitié (59,01 \%) et l'excellence (49,18 \%). Suivant notre analyse, ces internautes sont bien impliqués dans la promotion des valeurs olympiques et ils ont envie de les partager sur Internet car ils estiment qu'elles sont vraiment occultées au niveau institutionnel. Ils aimeraient de plus qu'elles soient valorisées sur ce dispositif pour sensibiliser l'ensemble des sportifs mais également un plus large public. Ces résultats sont de fait en cohérence avec ceux présentés précédemment. Les « fans » de la page « Promotion des valeurs olympiques» critiquent donc indirectement les sites institutionnels qui ne mettent pas en exergue ces valeurs qui devraient être pourtant d'après ces derniers les fondements de la gouvernance et de l'organisation des institutions sportives en général et olympiques en particulier.

Si notre démarche est essentiellement exploratoire à ce stade, il serait sans doute utile de convoquer au sein d'un dispositif méthodologique à la fois des techniques d'études qualitatives courantes (entretiens semi-directifs, analyse de matériaux) et d'initier de nouvelles approches qualitatives comme la netnographie ou l'ethnomarketing (Badot, Lemoine, 2010), ceci afin de mieux appréhender encore les discours et les usages des internautes.

4 Thèse de doctorat en sciences de l'information et de la communication, Université Nice Sophia-Antipolis, p. 216,377 p. 


\section{Conclusion}

Comme nous avons pu l'observer les membres de la communauté échangeant et animant la page Facebook proposée pour cette recherche sont impliqués dans la promotion des valeurs olympiques et désirent les partager via Internet. Cette page permet aux fans de s'exprimer librement. Ils expriment un désir pour la création d'un véritable espace virtuel pour s'exprimer, échanger et interagir avec les responsables du domaine sportif. Nous observons une critique indirecte des sites institutionnels qui ne promeuvent ces valeurs. De fait, même s'il existe de nombreux réseaux sociaux sportifs (réseaux multisports, spécialisés, professionnels ou plates-formes collaboratives) dans lesquels les fédérations sportives sont de plus en plus présentes comme elles le sont également sur des réseaux généralistes à l'instar de Facebook, les valeurs olympiques et leur valorisation, elles, le sont insuffisamment. Le succès spontané de la page créée sur Facebook semble témoigner du besoin d'un réel espace virtuel pouvant prendre la forme d'un réseau social ou s'adossant à un réseau existant qui permettrait de pouvoir échanger, promouvoir et diffuser les valeurs de l'olympisme.

À la lumière de ces réflexions et de ces premiers résultats nous pouvons considérer, en premier lieu, que la charte qui regroupe et s'inspire des valeurs de l'olympisme est l'archéotexte d'une organisation utopienne qui est présente dans l'esprit des sportifs et de leur public plus que dans les institutions censées en être le reflet. De fait, comme évoqué dans l'introduction l'utopie jouera ainsi son rôle de critique du modèle organisationnel et du secteur considéré.

En second lieu, cette controverse provoquée, prescrite et retraduite dans cette enquête exploratoire, trouve un bel écho dans les analyses d'Edgar Morin (2015) et de Michel Maffesoli (2015) et leurs critiques des grandes orientations normatives et visées rationalisantes de notre époque et de ses «bien-pensants ». Edgar Morin (2015 : 217) dans son œuvre et sa volonté à « reconnaître la complexité humaine et à mettre homo complexus au cœur de l'humanisme régénéré ; [comme quand il réaffirme avec lui] le premier principe humaniste qui est la reconnaissance de tout être humain, quel qu'il soit, d'où qu'il vienne, dans sa pleine humanité ». Le rationalisme étant promu comme valeur à la base de toute organisation et action, nous sommes bien dans une " "mauvaise" utopie [celle] qui veut réaliser l'harmonie et la perfection en éliminant tous les conflits et les perturbations" (ibid.: 118). Pour Edgar Morin encore (2015 : 118) : «La “bonne” utopie est irréalisable dans le présent, mais elle dispose des possibilités techniques ou pratiques de réalisation dans l'avenir : instituer un ordre international qui établirait la paix sur terre entre les nations, nourrir tous les habitants de la planète. Elle rejoint en cela la grande aspiration humaniste qui a toujours été considérée comme utopique et qu'il nous faut réassumer : réaliser un monde meilleur ». Ce que semble montrer aussi les réactions de nos répondants est ce besoin non de penser l'universel, mais de le vivre différemment dans 
ce que Michel Maffesoli (2015 : 147) nomme une « complétude proche [...] d'une vie vécue tant bien que mal, avec d'autres, en un lieu donné. L'utopie ne disparaît pas, elle prend une autre forme ». En ce sens il renvoie, avec Albert Camus, aux " utopies relatives ». Pour lui (ibid. 148) : "l'utopie "relative" [qu'il qualifie aussi de populaire] redonne à la vie quotidienne une indéniable noblesse ; cela, également, met l'accent sur la relation. C'est cette utopie, qui relie et qui redonne de la confiance, qui est le défi lancé par la sociabilité de l'homme sans qualité aux divers protagonistes de l'action publique, les politiques en particulier».

\section{BIBLIOGRAPHIE}

ATTALI Michaël et al. (2009), Les valeurs de l’olympisme. Un modèle éducatif en débat, Paris, Éd. L'Harmattan.

BADOT Olivier et LEMOINE Jean-François (2010), « L'ethnomarketing au service de la prospective : une application au secteur de la distribution ", Management $\&$ Avenir, $n^{\circ} 19$, p. $37-47$.

BOLI Claude (2009), "Les Jeux Olympiques, fierté nationale et enjeu mondial », Géménos, Éd. AFRAPS, p. 59-67.

CAMPANELLA Tommaso (2000), La cité du soleil, Paris, Éd. Mille et une nuits (traduction par Arnaud Tripet) de lédition originale écrite en 1623 (CivitasSolis).

CHATZIEFSTATHIOU Dikaia (2005), «The Changing Nature of the Ideology of Olympism in the Modern Olympic Era». A Doctoral Thesis Submitted in partial fulfilment of the requirements for the award of Doctor of Philosophy of Loughborough University, $\mathrm{p} 15$.

CHOUQUER Gérard et DAUMAS Jean Claude (2006), « Autour de Ledoux : architecture, ville et utopie ", actes du colloque à la Saline royale d'Arc-et-Senans, Les Cahiers de la MSHE Ledoux, n 13, Presses Universitaires de Franche-Comté.

COAKLEY Jay (2001), Sport in Society: Issues and Controversies, 7th ed, New York, McGraw-Hill.

COUBERTIN Pierre (1935), "Mémoires olympiques ", Bureau international de pédagogie sportive, Lausanne.

CORALL Durántez Conrado et al. (2010), «Principles of The Olympic Movement», Journal of Human Sport and Exercise, vol. V, n ${ }^{\circ} 1$, p. 3-14.

DEBOUT Simone (1978), L'Utopie de Charles Fourier, Paris, Éd. Payot.

DIANA Jean-François et MEYER Vincent (2004), « Dire le dopage. Les enjeux sociologiques et médiatiques ", Questions de communication, Série actes 1, Nancy, Presses universitaires de Nancy.

HOBERMAN John (1986), The Olympic Crisis. Sport, Politics and the Moral Order, New Rochelle, NY: Caratzas Publishing Co, Inc.

LATOUR Bruno (2007), "La cartographie des controverses ", Technology Review, $\mathrm{N}^{\circ}$ 0, p. 82-83. 
LIOTARD Philippe (2004), " l'éthique sportive, une morale de la soumission ? ", p. 117-156, in Michaël Attali, Le sport et ses valeurs, Paris, Éd. La Dispute.

MAFFESOLI Michel et STROHL Hélène (2015), Les nouveaux bien-pensants, Paris, Éd. du moment.

MALHOTRA Naresh K. (2004), Review of Marketing Research, Volume 1, Routledge. MONTANDONB et al. (1987), Entre parents et enseignants : un dialogue impossible? Berne, Éd. Peter Lang.

MORE Thomas (1518), L'Utopie, fac-similé du texte latin de l'édition de Bâle, traduction, présentation et commentaires par André Prévost, Mame, 1978.

MORIN Edgar (2015), L'aventure de la méthode, Paris, Éd. Du Seuil.

MULLIEZ Jacques (2013), Thomas More (1478-1535) : Au risque de la conscience, Bruyères-le-Châtel (Essonne), Éd. Nouvelle Cité.

MUSSO Pierre (1999), Saint-Simon et le saint-simonisme, Paris, Presses universitaires de France.

OUERGLI Faika (2014), La promotion des valeurs olympiques au sein des communautés virtuelles : le cas de Facebook, Thèse en sciences de l'information et de la communication, Université Nice Sophia Antipolis.

RASSE Paul (2003), « Utopies de la Cité de la sagesse », in L'Héritage d'une utopie : essai sur la communication et l'organisation de Sophia-Antipolis, dir. Araszkiewiez J., Edisud, p. 11-23.

SEAGRAVE Jeffrey et CHUD Donald (1981), Olympism, Champaign, Illinois : Human Kinetics, Champaign, III., 1988, p. 149-161.

SFEZ Lucien (2000), «Utopie I, La fabrique de l'utopie », Quaderni, n 40, préface.

SIMSON Vyv and JENNINGS Andrew (1992), The Lords of the Rings. Power, Money and Drugs in the Modern Olympics, London: Simon and Schuster Ltd. First Edition, 288 p.

Résumé: La réflexion que nous menons part du constat suivant : les organisations sportives peinent à afficher des références aux valeurs olympiques et des communautés virtuelles en débattent et s'y substituent. En effet, les spectateurs du monde sportif par le biais des réseaux sociaux véhiculent ces valeurs que l'on devrait (re)trouver au cœur des interactions numériques entre pratiquants et institutions sportives.

Nos propos sont structurés comme suit : après avoir rappelé les fondements de l'olympisme, nous tenterons de montrer que les valeurs associées à l'olympisme - censées représenter " la colonne vertébrale », non seulement de l'organisation olympique, mais de toute institution sportive - peuvent être considérées, en première analyse, comme les facettes de l'archétype d'une organisation utopienne. À la suite, après avoir exposé quelques faits qui témoignent de la controverse évoquée, nous présenterons en seconde analyse, celle de certains sites institutionnels en lien avec le comité international olympique couplée aux résultats d'une enquête par questionnaire menée auprès d'une communauté développée sur Facebook autour d'une page intitulée «Promotion des valeurs olympiques » composée de personnes ayant une expérience professionnelle dans le domaine sportif. En conclusion, nous reviendrons sur le besoin de «bonne utopie » 
(Morin 2015) que, sans doute, les organisations comme les institutions (i.e. celles et ceux qui les représentent) peinent de plus en plus à traduire et qui ne se retrouvent plus, tout à fait, dans les grands discours ou dans une ambition à penser l'universel ouvrant in fine comme le suggère Michel Maffesoli (2015) sur une « normopathie ».

Mots-clés : valeurs de l'olympisme, utopie, communautés virtuelles, IOC, institutions sportives, Facebook.

\begin{abstract}
The reflection we are conducting share paradox sports organizations struggle to see references to the Olympic values and virtual communities discuss and superseded. Indeed, spectators of the sports world through social networks convey these that should be (re) at the heart of digital interaction between practitioners and sporting institutions.

Our comments are structured as follows: after recalling the foundations of Olympism, we will attempt to show that the values associated with the Olympic spirit-supposed to represent the "backbone", not only of the Olympic organization, but any institution sports-can be considered, in the first analysis, as facets of the archetype of a utopian organization. As a result, after stating some facts that attest to the controversy raised, we will present in the second analysis, that of some institutional sites in conjunction with the International Olympic Committee coupled with the results of a questionnaire survey conducted among a developed community on Facebook over a page entitled "Promotion of Olympic Values» composed of persons who have professional experience in sports. In conclusion, we will return to this need for "good utopia» (Morin 2015), no doubt, organizations such as the institutions (those who represent them) are struggling increasingly to be translated and which are not found more, quite in the rhetoric or an ambition to think the universal opening in fine as Michel Maffesoli (2015) suggests a "normopathie».
\end{abstract}

Keyword: Olympic values, utopia, virtual community, IOC, Sports Institutions, Facebook.

\title{
Webographie
}

http://www.unige.ch/fapse/SSE/teachers/perrenoud/php_main/ php_1987/1987_01.html

http://www.olympic.org/fr/cio

http://www.olympic.org/fr/charte-olympique/documents-rapports-etudespublications

http://olympicmuseum.de/charter/olympiccharter (Sept2004.pdf) http://www.tropes.fr/ManuelDeTropesVF810.pdf 
Annexe 1 : analyse des sites apparaissant en premier sur Google avec les motsclés, "l les valeurs olympiques"

\begin{tabular}{|c|c|c|c|}
\hline $\begin{array}{l}\text { Le classement } \\
\text { des sites }\end{array}$ & $\begin{array}{c}\text { Les caractéristiques } \\
\text { des sites }\end{array}$ & $\begin{array}{c}\text { Visualisation des valeurs } \\
\text { olympiques }\end{array}$ & Commentaires \\
\hline 1) $\mathrm{FO}^{5}$ & $\begin{array}{l}\text {-Non interactif } \\
\text {-Site statique } \\
\text {-Facilité d'usage } \\
\text {-Facilité de } \\
\text { navigation } \\
\text {-Le contenu est } \\
\text { bâti sur } \\
\text { des images sportives }\end{array}$ & $\begin{array}{l}\text { - C'est une brochure } \\
\text { d'enfants. } \\
\text { - L'icône «Rechercher» } \\
\text { placée en haut de page } \\
\text { d'accueil de la zone de } \\
\text { saisie. Un fichier sous } \\
\text { formats PDF mentionne } \\
\text { ces valeurs dans le tiers } \\
\text { de la page d'une façon } \\
\text { brève avec la présence } \\
\text { de quelques sponsors } \\
\text { comme Adidas, EDF, } \\
\text { SOMFY,...etc. }\end{array}$ & $\begin{array}{l}\text { - C'est le premier site } \\
\text { qui apparaît et qui } \\
\text { n'est pas destiné aux } \\
\text { valeurs olympiques. En } \\
\text { effet, sa vocation est de } \\
\text { promouvoir une ville } \\
\text { Française lors des JO } \\
\text { d'hivers. } \\
\text { - Ce site promotionnel } \\
\text { est plutôt dédié aux } \\
\text { adultes et non pas aux } \\
\text { enfants. On pourra le } \\
\text { constater avec notre } \\
\text { étude qu'il y a un } \\
\text { décalage avec le profil de } \\
\text { nos fans. }\end{array}$ \\
\hline 2) $\mathrm{Blog}^{6}$ & $\begin{array}{l}\text {-Non interactif } \\
\text {-Site statique } \\
\text {-Absence d'images } \\
\text { ou photos sportives } \\
\text { - Facilité d'usage } \\
\text {-Facilité de } \\
\text { navigation }\end{array}$ & $\begin{array}{l}\text { Ce blog est consacré aux } \\
\text { valeurs olympiques qui } \\
\text { sont bien développées } \\
\text { et expliquées. Pour les } \\
\text { mettre en valeur, l'auteur } \\
\text { les a mis dans un tableau } \\
\text { symbolique intitulé« On } \\
\text { the board to day @๖ les } \\
\text { valeurs olympiques». }\end{array}$ & $\begin{array}{l}\text {-Une certaine similitude } \\
\text { entre ce blog et notre } \\
\text { étude de recherche, } \\
\text { mais tous les fans de } \\
\text { la page Facebook que } \\
\text { nous avons créée } \\
\text { n'ont pas connaissance de } \\
\text { ce site. }\end{array}$ \\
\hline $\begin{array}{l}\text { 3) Olympic } \\
\text { Torch Relay }^{7}\end{array}$ & - Fichier PDF & $\begin{array}{l}\text { - Ce fichier expose les } \\
\text { valeurs olympiques } \\
\text { incarnées par le } \\
\text { TorchRelay, à savoir : } \\
\text { unité, participation, le } \\
\text { passé et l'avenir. } \\
\text {-L'aspect humain et une } \\
\text { attitude positive sont mis } \\
\text { en avant. }\end{array}$ & $\begin{array}{l}\text { - Ce document est en } \\
\text { relation avec notre thème } \\
\text { de recherche. }\end{array}$ \\
\hline
\end{tabular}

5 Site France Olympique http://franceolympique.com/files/File/Annecy_2018/Brochure\%20Enfants\%20Annecy\%20208.pdf 6 Blog « l'actualité du Marketing sportif », 7 http://www.awbb.be/transi/jeux\%20olympiques.pdf 


\begin{tabular}{|c|c|c|c|}
\hline $\begin{array}{l}\text { Le classement } \\
\text { des sites }\end{array}$ & $\begin{array}{c}\text { Les caractéristiques } \\
\text { des sites }\end{array}$ & $\begin{array}{c}\text { Visualisation des valeurs } \\
\text { olympiques }\end{array}$ & Commentaires \\
\hline 4) $\mathrm{COIB}^{8}$ & $\begin{array}{l}\text { - Non interactif et } \\
\text { non attractif } \\
\text { - Site statique } \\
\text { - Facilité d'usage } \\
\text { - Facilité de } \\
\text { navigation } \\
\text { - Le logo du COIB } \\
\text { est l'alliance du } \\
\text { drapeau belge } \\
\text { avec le symbole } \\
\text { olympique qui se } \\
\text { trouve dans la page } \\
\text { d'accueil. } \\
\text { - La flamme } \\
\text { olympique est mise } \\
\text { en relief dans le } \\
\text { haut de la page } \\
\text { d'accueil. }\end{array}$ & $\begin{array}{l}\text { - Les valeurs olympiques } \\
\text { sont proposées } \\
\text { explicitement sous la } \\
\text { rubrique «olympisme» } \\
\text { dans la page d'accueil. }\end{array}$ & $\begin{array}{l}\text { - La visualisation des } \\
\text { valeurs Olympiques nest } \\
\text { pas immédiate. }\end{array}$ \\
\hline
\end{tabular}

Annexe 2 : analyse des sites apparaissant en premier sur Google avec les motsclés, "les valeurs de l'olympisme "

\begin{tabular}{|l|l|l|l|}
\hline $\begin{array}{c}\text { Le classement } \\
\text { des sites }\end{array}$ & $\begin{array}{c}\text { Les caractéristiques des } \\
\text { sites }\end{array}$ & $\begin{array}{l}\text { Visualisation des valeurs de } \\
\text { l'olympisme }\end{array}$ & \multicolumn{1}{c|}{ Commentaires } \\
\hline 1) $\mathrm{F} \mathrm{O}^{9}$ & $\begin{array}{l}\text { - Brochure en PDF } \\
\text {-Des informations } \\
\text { liées au mouvement } \\
\text { olympique sont dans le } \\
\text { contenu. }\end{array}$ & $\begin{array}{l}\text {-Les valeurs olympiques } \\
\text { sont repérées dans } \\
\text { une brochure avec } \\
\text { notamment les principes } \\
\text { fondamentaux de } \\
\text { l'olympisme et de la charte } \\
\text { olympique. }\end{array}$ & $\begin{array}{l}\text { - Le site n'est pas } \\
\text { adapté au cour de } \\
\text { cible .Il est en décalage } \\
\text { avec l'ambiance } \\
\text { et l'image du CIO. }\end{array}$ \\
\hline 2) & $\begin{array}{l}\text { - Cette encyclopédie } \\
\text { libre spécifie uniquement } \\
\text { le mouvement } \\
\text { olympique mais les } \\
\text { valeurs olympiques } \\
\text { sont inexistantes. }\end{array}$ & $\begin{array}{l}\text { - Absence des } \\
\text { valeurs olympiques. }\end{array}$ \\
\hline
\end{tabular}

8 Comité Olympique et Interfédéral Belge, http://www.awbb.be/transi/jeux\%20olympiques.pdf 9 http://bouchesdurhone.franceolympique.com/bouchesdurhone/fichiers/File/TREIZ_OLYMPIQUE/N37/13b.pdf 10 http://fr.wikipedia.org/wiki/Jeux_olympiques 


\begin{tabular}{|c|c|c|c|}
\hline $\begin{array}{c}\text { Le classement } \\
\text { des sites }\end{array}$ & $\begin{array}{c}\text { Les caractéristiques des } \\
\text { sites }\end{array}$ & $\begin{array}{c}\text { Visualisation des valeurs de } \\
\text { l'olympisme }\end{array}$ & Commentaires \\
\hline $\begin{array}{l}\text { 3) Les JO en } \\
\text { Chine } 2008^{11}\end{array}$ & $\begin{array}{l}\text { - Non interactif et non } \\
\text { attractif. } \\
\text { - Site statique. } \\
\text { - L'image de la charte } \\
\text { olympique est incluse } \\
\text { dans la page d'accueil. } \\
\text { - Le contenu du } \\
\text { site est centré sur la } \\
\text { charte olympique } \\
\text { et les principes } \\
\text { fondamentaux de } \\
\text { lolympisme. } \\
\text { - Dans le bas de page, } \\
\text { un lien de «CIO» } \\
\text { pointe vers le site du } \\
\text { Comité International } \\
\text { Olympique contenant } \\
\text { toutes les informations. }\end{array}$ & \begin{tabular}{|l|} 
- Ce site contient \\
des informations \\
exclusivement sur le \\
mouvement olympique \\
et les valeurs olympiques \\
sont sous représentées.
\end{tabular} & $\begin{array}{l}\text { - Le site ne reflète pas } \\
\text { l'ambiance des JO }\end{array}$ \\
\hline $\begin{array}{l}\text { 4) Site } \\
\text { Français } \\
\text { intitule } \\
\text { «allons en } \\
\text { France» }\end{array}$ & $\begin{array}{l}\text { - Site interactif dans } \\
\text { la mesure où l'on peut } \\
\text { poster nos témoignages } \\
\text { sur les valeurs } \\
\text { olympiques. } \\
\text { - Les logos présentent } \\
\text { les différentes } \\
\text { disciplines comme la } \\
\text { natation, le football, le } \\
\text { handball...etc. }\end{array}$ & $\begin{array}{l}\text { - Le contenu du site } \\
\text { est placé en accent sur } \\
\text { le thème des valeurs } \\
\text { olympiques. }\end{array}$ & $\begin{array}{l}\text { - Il s'agit du site } \\
\text { qui serait le plus } \\
\text { en phase avec une } \\
\text { population qui a un } \\
\text { rôle de prescripteur } \\
\text { et qui correspond à } \\
\text { notre page Facebook } \\
\text { «Promotion des valeurs } \\
\text { olympiques». }\end{array}$ \\
\hline
\end{tabular}

$11 \mathrm{http} / / /$ fr.beijing2008.cn/spirit/symbols/charter/

$12 \mathrm{http} / / /$ www.allonsenfrance.com/2011/spip.php?article140 
Annexe 3 : le profil des fans consulté le 10 Mars 2013

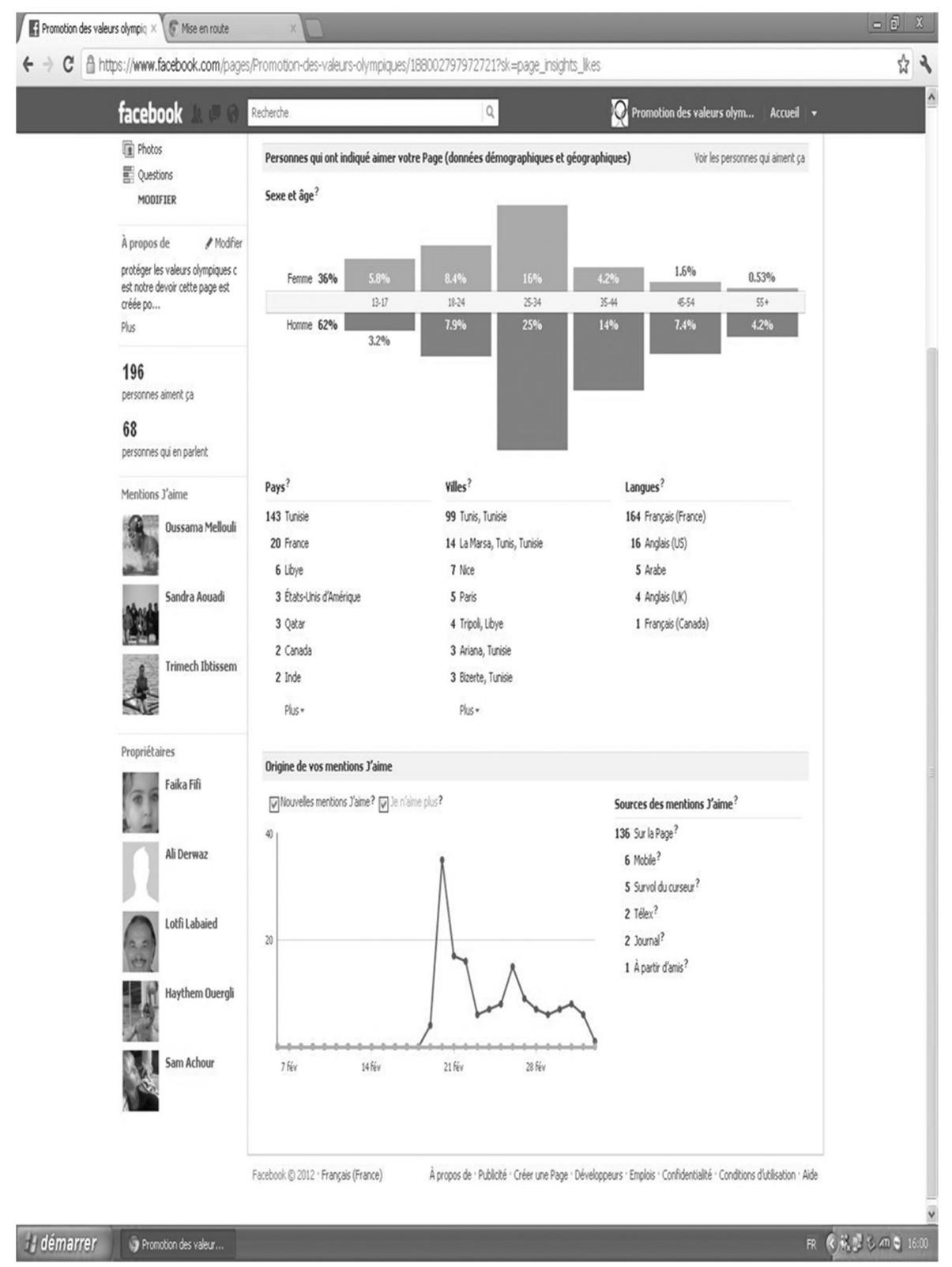


